\title{
An Energy-Stabilized Varied-Line-Space-Monochromator Undulator Beam Line for PEEM Illumination and Magnetic Circular Dichroism
}

\author{
Tony Warwick, Wayne McKinney, Ed Domning, Andrew Doran and Howard \\ Padmore
}

Advanced Light Source, Lawrence Berkeley National Laboratory, Berkeley, CA94720

\begin{abstract}
A new undulator beam line has been built and commissioned at the Advanced Light Source for illumination of the PEEM3 microscope. The beam line delivers high flux beams over an energy range from $\mathrm{C} 1 \mathrm{~s}$ through the transition metals to include the $\mathrm{M}$ edges of the magnetic rare earth elements. We present details of the optical design, and data on the performance of the zero-order tracking of the photon energy.
\end{abstract}

Keywords: synchrotron beamline monochromator varied line space grating PEEM

PACS: 07.85.Qe

\section{BEAM LINE DESIGN}

A new undulator beam line has been built and commissioned at the Advanced Light Source, using a 50mm period Elliptically Polarizing Undulator with 1.9Gev electrons as a source for Photo Electron Emission Microscopy (PEEM) and Magnetic Circular Dichroism (MCD). The beam line delivers high flux beams over an energy range from $\mathrm{C} 1 \mathrm{~s}$ through the transition metals to include the $\mathrm{M}$ edges of the magnetic rare earth elements. This energy range $(200 \mathrm{eV}$ to $1800 \mathrm{eV})$ is provided with full polarization control, with any orientation of the E vector, linear or circular/elliptical.

The beam line employs a triple-ruled varied-line-space grating monochromator at moderate spectral resolution $(\mathrm{R} \cong 3000)$. The plane grating was polished and ruled by Carl Zeiss Laser Optics. The monochromator also provides a focused zero-order beam that is monitored for its position relative to the exit slit to provide precisely reproducible energy scans. The spectral resolution required for MCD is modest but the measurements often require the subtraction of successive energy scans. These must have precisely identical energy scales to minimize the noise in the resulting MCD spectrum. This reproducibility will be accomplished by a digital recording of the image of the focused zero-order position, at each value of the photon energy through a spectral scan. Repeat scans are to be made under digital servo-control to replicate the energy scale at the level of 1 part in $10^{6}[1]$.

Computer-controlled micro-focus mirrors are included to illuminate a field of a few millimeters on the sample surface, for low magnification microscopy. For high magnification microscopy these mirrors can focus and steer the $\mathrm{x}$-ray beam to illuminate a $10 \mu \mathrm{m}$ field of view.

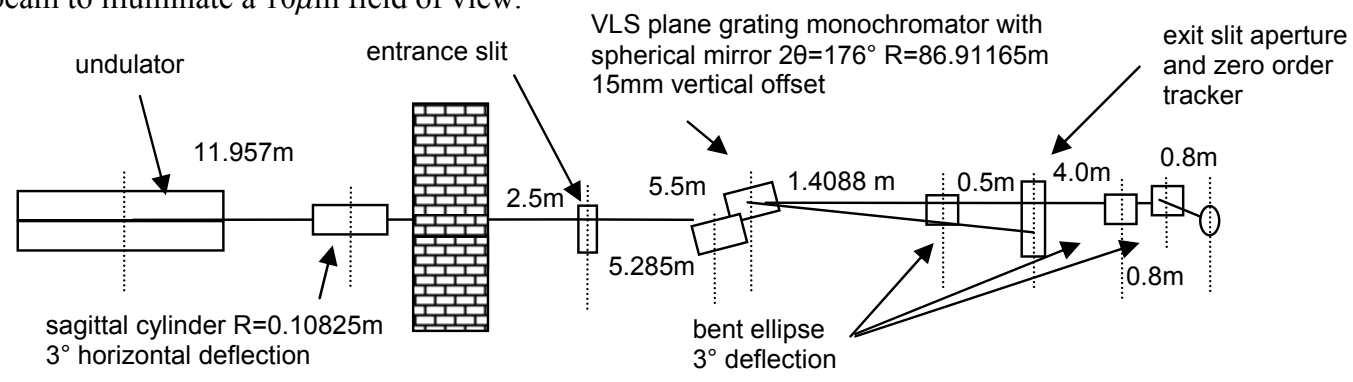

FIGURE 1. Beam line layout (side view) 


\section{Simple Representation of the Monochromator Optical Scheme}

The monochromator consists of a spherical mirror followed by a plane varied-line-space grating [2]. The following sketch illustrates the optical layout.

$\mathrm{n}(\mathrm{w})$ is the groove number counted from the center $(\mathrm{w}=0)$ with $\mathrm{n}(\mathrm{w}=0)=0 . \quad \mathrm{m}$ is positive for diffraction inside the zero order, closer to the grating normal. $\alpha$ and $\beta$ are the usual incident and diffracted angles measured at $\mathrm{w}=0$, with $\alpha$ positive and $\beta$ negative. Considering only linear variation of the groove density, the groove density is given (Shadow convention [3]) by:

$$
\frac{\partial n}{\partial w}=(N+b w)
$$

where $N$ is the central groove density and $b$ specifies the variation of the

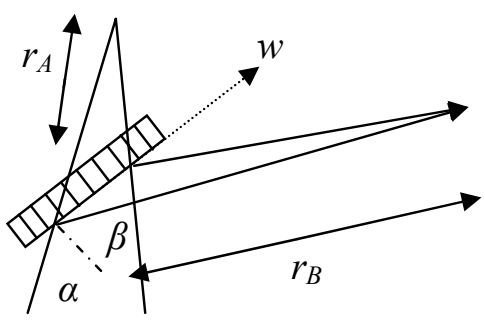
line spacing. The derivative of the optical path function must be zero:

$$
\frac{\partial F}{\partial w}=-\sin \alpha-\sin \beta+w\left(\frac{\cos ^{2} \alpha}{r_{A}}+\frac{\cos ^{2} \beta}{r_{B}}\right)+m \lambda(N+b w)=0
$$

Combining this with the grating equation $(\sin \alpha+\sin \beta=N m \lambda)$ gives:

$$
\frac{\cos ^{2} \alpha}{r_{A}}+\frac{\cos ^{2} \beta}{r_{B}}+b m \lambda=0
$$

Since $\alpha$ and $\beta$ vary as the monochromator is scanned, this last equation can be satisfied at only one wavelength, which is where the instrument is in focus. With that value for $b$ the focus is approximately maintained across a range of wavelengths and the loss of resolution within this range is normally very small. Higher order terms in the variation of the line spacing can be introduced. The quadratic term can compensate for the coma aberration of the spherical mirror (which is quadratic in the mirror aperture coordinate). The necessity for this correction depends on the size of the illuminated area of the mirror. As a practical procedure, once the linear term in the variation of the line spacing is determined, a ray-trace (Shadow) with realistic illumination allows one to tune the quadratic term, for optimum spectral resolution.

\section{Energy Resolution}

Figure 2 shows the canonical resolution test for soft $\mathrm{x}-$ ray beamlines. This beam line is capable of a resolving power up to $\mathrm{R}=5,000$, using $15 \mu \mathrm{m}$ entrance slits and $30 \mu \mathrm{m}$ exit slits, here, at the low-energy end of the middle grating (500 lines/mm). At this low-energy end of the operational range the resolution is limited by residual defocus, after correction by the varied line spacing. At the high energy end of the range, $900 \mathrm{eV}$ for this grating, the resolution is limited by the polished tangential slope errors of the two monochromator optics $(0.5 \mu \mathrm{rad} \mathrm{rms})$.

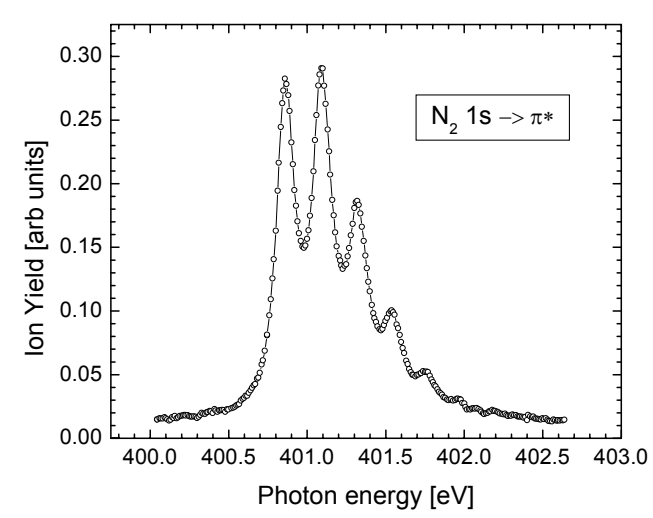

FIGURE 2. Vibrationally resolved N2 absorption spectrum with $\mathrm{R} \approx 5,000$

\section{Zero-Order Tracking}

A carefully engineered exit-slit assembly carries a detector to monitor the height of the focused zero-order below the exit slit. The vertical separation of the zero order from the exit slit will eventually be a high fidelity measurement 


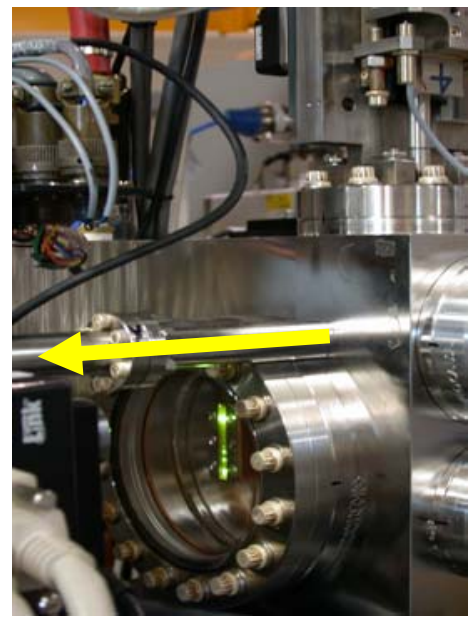

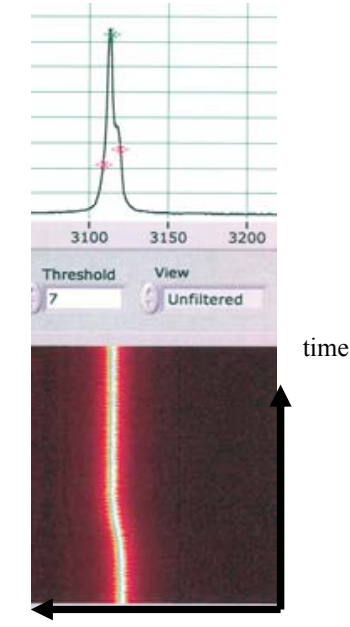

vertical position on YAG
FIGURE 3. Exit slit assembly at beam line 11.0.1. The monochromatic x-ray beam (yellow arrow) passes to the experiment. Zero order is visible on a YAG screen in front of the microscope, along with unused undulator harmonics in first order. The image vertical line-scan history is shown on the right, capturing the monochromator as it steps $0.5 \mathrm{eV}$ at $750 \mathrm{eV}$. Ambient vibrations can be seen corresponding to about $1 / 20,000$ of the photon energy. The vertical-line-scan centroid is determined with a precision $1 / 100,000$ of the photon energy after 1 second of averaging.

of the energy selected by the diffraction grating. It is a measurement largely unaffected by thermal motion of the entrance slit, of the exit slit assembly, or by thermal changes of the grating angle. The initial detection scheme consists of a microscope viewing the focused zero-order on a single crystal YAG scintillator. The microscope objective is carried on a temperature stabilized $\left(0.1^{\circ} \mathrm{C}\right)$ mount. Eventually it will need to be held to about $10 \mathrm{~nm}$ between successive scans if the ultimate precision ( 1 part in $10^{6}$ of the photon energy) of monitoring is to be achieved. The thermal properties of this detector mount are not yet properly implemented or studied.

This ultimate precision may require an improved detection scheme, but this visual detector has been essential to understand the behavior of the monochromator during beam line commissioning. The vertical centroid of the focused zero-order can be determined to $1 / 10$ pixel between steps of an energy scan. So at present the precision of photon energy monitoring is about $10^{-5}$ during a scan. A factor 10 improvement in precision is desirable, and the accuracy must reach the same level. The current detection scheme has the image of the zero-order focal spot moving vertically on the camera chip [4] as the monochromator scans. Pixel-to-pixel gain variations show up as sub-pixel glitches in the motion of the vertical centroid of the image, but these are the same from scan to scan. One important advantage of the present scheme is that there is no (deliberate) motion of the detector during measurement. In the future, a zeroing knife-edge sensor may be adopted, but that would involve detector motion during the scan that would need to be accurately encoded, to tens of nm.

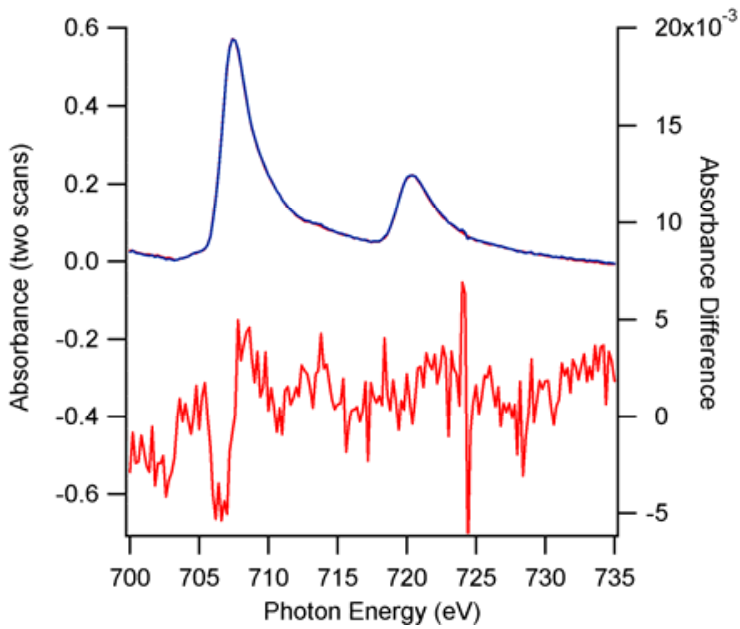

FIGURE 4. Two successive absorbance measurements from the same Fe film (arbitrary units, superimposed) and the difference.

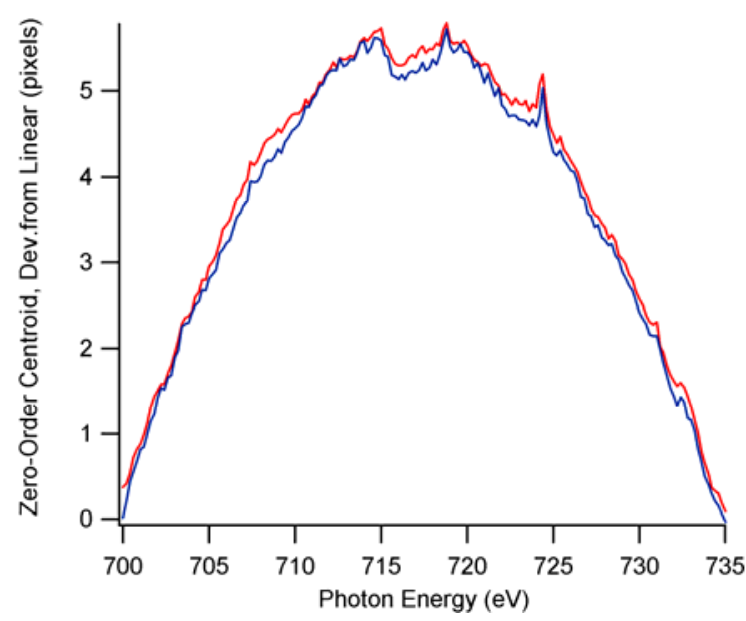

FIGURE 5. Centroid motion of the zero-order, during the two absorbance scans. The linear variation is subtracted. Glitches common to both scans are pixel-gain effects. Systematic differences between the scans are probably due to thermal motion of the detector and need further study. 
Figure 4 shows the first test measurement. Two successive NEXAFS spectra were measured through an Fe film $26 \mathrm{~nm}$ thick, into a silicon-diode detector. These absorption spectra should be identical. Small differences between the two scans arise due to decay of the stored electron beam and the drift of the beam line focus at the monochromator entrance slit. These effects were normalized using the integrated intensity of the zero-order light from the zero-order tracker. The results show good reproducibility of the monochromator, without any adjustment of the energy scale based on the zero-order centroid data. The noise in the difference of the two scans (Fig. 4) is dominated by residual intensity fluctuations. This is apparent because the noise is not localized to the regions of the spectrum with peaks. Less noisy normalization must be pursued before the effects of energy-scale differences can be addressed.

The deviation from linearity of the zero order centroid is shown in Fig. 5 for the two successive scans. Systematic differences of the order 0.2 pixels are seen above the noise, corresponding to 1:50,000 change of the energy scale, which is apparently the present limit of the accuracy of the instrument At this level we are sensitive to thermal expansion of about $1 \mu \mathrm{m}$.. Further study of temperature effects is required.

It should be noted that optical microscopy using centroiding techniques to determine the position of a fluorophore label on a biological molecule has been shown to be capable of localization to $<1 \%$ of one sigma optical resolution [5]. Following the methodology of Broboff [6] who analyzed the intrinsic centroiding accuracy limited by Poisson statistics, it can be shown that for the levels of signal available in the zero order tracker, a centroiding accuracy of $<1 \%$ of 1 pixel at $10 \mathrm{~Hz}$ should be readily achievable. As the zero order scans over the pixel array, to get a precision of this level will require the use of the same sophisticated calibration and normalization techniques used in centroiding optical microscopy.

\section{CONCLUSIONS}

A new undulator beamline for PEEM illumination and Magnetic Circular Dichroism is operational at the Advanced Light Source. This beamline now delivers $5 \times 10^{11}$ photons per second at a resolving power $\mathrm{R}=3,000$ into an illuminated area as small as 15 microns, for PEEM. Spectral tests for MCD show an MCD noise level of the order $10^{-3}$ using simple normalization techniques. Additional instrumentation is under development to stabilize the energy scale. This involves ongoing thermal stability studies of the zero-order tracker, detector development, and the implementation of low noise normalization schemes. The function of the zero-order-tracker is demonstrated and we are working to reduce the intrinsic beam line MCD noise by a factor of ten.

\section{ACKNOWLEDGMENTS}

This work was supported by the Director, Office of Energy Research, Office of Basic Energy Sciences, and Materials Sciences Division of the U.S. Department of Energy, under Contract No. DE-AC03-76SF00098.

\section{REFERENCES}

1. J. Feng, T. Warwick and H. Padmore "Energy stability requirement of PEEM3 beam line and related angular resolution of the monochromator" ALS internal report, April 2003

2. K Amemiya, Y. Kitajima, T. Ohta and K. Ito "Design of a Holographically Recorded Plane Grating with a Varied Line Spacing for a Soft X-Ray Grazing Incidence Monochromator” J. Synchrotron Radiation 3 (1996) 282-388

C. F. Hague, J. H. Underwood, A. Avila, R. Delaunay, H. Ringuenet, M. Marsi, and M. Sacchi "Plane-grating flat-field soft X-ray spectrometer " Rev. Sci. Instrum. 76 (2005) 023110

M. C. Hettrick, J. H. Underwood, P. J. Batson and M J. Eckart "Resolving power of 35,000 (5 mA) in the extreme ultraviolet employing a grazing incidence spectrometer" Appl. Opt. 27 (1988) 200

3. C. Welnak, P. Anderson, M. Khan, S. Singh, and F. Cerrina "Recent developments in SHADOW" Rev. Sci. Instrum. 63 (1992) 865-868 and see http://www.esrf.fr/computing/scientific/raytracing/

4. DALSA "Piranah II" digital line scan camera.

5. A. Yildiz, et al. "Myosin V Walks Hand-Over-Hand: Single Fluorophore Imaging with 1.5-nm Localization" Science 300 (2003) 2061-2065

6. N. Bobroff "Position measurement with a resolution and noise-limited instrument. Rev. Sci. Instr. 57 (1986) 1152-1157 